\title{
Noncoalescence in the Oblique Collision of Fluid Jets
}

\author{
Navish Wadhwa, ${ }^{1, *}$ Pavlos Vlachos, ${ }^{2}$ and Sunghwan Jung ${ }^{1, \dagger}$ \\ ${ }^{1}$ Department of Engineering Science and Mechanics, Virginia Tech, Blacksburg, Virginia 24060, USA \\ ${ }^{2}$ Department of Mechanical Engineering, Virginia Tech, Blacksburg, Virginia 24060, USA \\ (Received 31 October 2012; revised manuscript received 8 February 2013; published 20 March 2013)
}

\begin{abstract}
When two jets of fluid collide, they can "bounce" off each other, due to a thin film of air which keeps them separated. We describe the phenomenon of stable noncoalescence between two jets of the same fluid, colliding obliquely with each other. Using a simple experimental setup, we carry out a parametric study of the bouncing jets by varying the jet diameter, velocity, angle of inclination, and fluid viscosity, which suggests that the contact time of bouncing jets scales as the square root of the normal Weber number We. A dimensionless parameter $K=(\mathrm{We} \sqrt{\operatorname{Re}} / \sin \alpha)^{1 / 2}$, where $\operatorname{Re}$ is the normal Reynolds number and $\alpha$ the angle of inclination of the jets, quantitatively captures the transition of colliding jets from bouncing to coalescence. This parameter draws parallels between jet coalescence and droplet splashing and indicates that the transition is governed by a surface instability. Stable and continuous noncoalescence between fluid jets makes it a good platform for experimental studies of the interaction between fluid interfaces and the properties of the interfacial air films.
\end{abstract}

DOI: 10.1103/PhysRevLett.110.124502

PACS numbers: 47.55.df, 47.15.- $-\mathrm{x}, 47.55 . \mathrm{N}-$

While intuition tells us that two streams of fluid colliding with each other will mix together into one stream, that is not always the case. Contrary to the expectation, two jets of fluid can undergo stable noncoalescence and thus bounce off each other upon collision [Fig. 1(a)]. Understanding the interaction between two fluid interfaces is central to a variety of industrial and everyday processes, such as raindrop formation [1], spray coating [2], inkjet printing [3], and metallurgical processes [4]. In 1878, Lord Rayleigh $[5,6]$ was the first to point out that drops and jets could bounce off each other, which led to a series of investigations on the noncoalescence between drops and jets [7-10]. Noncoalescence between a drop and the free surface of fluid was first reported by Reynolds in 1881 [11] and has since been studied in much detail [12-14]. Since then, many variations of drop-surface system have been explored, such as drop bouncing on an oscillating surface [15], drop bouncing on a soap film [16,17], and drop floating on a hydraulic jump [18]. The jets noncoalescence problem was revisited by Wadhwa and Jung [19], who reported noncoalescence between two and three jets and between a jet and drops of the same fluid. However, there have still been no quantitative studies on the noncoalescence in jets since the early work by Rayleigh.

Noncoalescence between two drops, or between a drop and a surface, takes place because air takes a finite time to flow out of the region between the two interfaces when they come close to each other. As soon as the air is drained out from between the interfaces, coalescence takes place. A fundamental difference between the drop noncoalescence and jet noncoalescence, is that the latter is a steady state process; the air film separating the two jets is entrained by the flow of the jets and is continuously replenished, resulting in self-sustained continuous noncoalescence.
Figure 1(a) shows an example of two silicone oil jets bouncing off each other upon collision. The jets drag along air into the collision region where it is squeezed in a thin film. Since the thickness of the air film is much smaller than the other dimensions, lubrication approximation is applicable [20], which results in high magnitude forces keeping the jets apart. When the jet velocity $V$ is increased beyond a threshold, the jets undergo coalescence, as shown in Fig. 1(b).

Silicone oils (Clearco Products Co., Inc.) are used for most experiments in order to avoid any surface contamination. These oils have kinematic viscosity $\nu=4.37,8.52$, 17.9, and $46.6 \mathrm{cSt}$, surface tension $\sigma=19.7,20.1,20.6$, and $20.8 \mathrm{mN} / \mathrm{m}$, and density $\rho=918,935,950$, and $960 \mathrm{~kg} / \mathrm{m}^{3}$, respectively. A few experiments with glycerol solution in water $(75 \%$ by weight, $\sigma=66.1 \mathrm{mN} / \mathrm{m}$, $\nu=22.44 \mathrm{cSt}$, and $\left.\rho=1195 \mathrm{~kg} / \mathrm{m}^{3}\right)$ are conducted to explore the role of surface tension. Two blunt tip stainless steel needles with a circular cross section are fitted on three-axis translation stages and arranged such that the jets emerging from them lie in the same vertical plane and collide with each other obliquely. Needles with internal diameter $D=0.58,0.65,0.81,0.97$, and $1.35 \mathrm{~mm}$ are used in this study. The needles are supplied with fluid from two glass syringes using a syringe pump, whose flow rates could be precisely adjusted to control the velocity of the colliding jets. Flow rates ranging from 400 to $2900 \mathrm{ml} / \mathrm{hr}$ are used in the experiments. The collision region is imaged using a digital SLR camera, from a direction perpendicular to the plane containing the jets.

We measure the contact length $L$ and the jet angle $\alpha$ of bouncing jets from the images for various combinations of $V$ and $D$ for different fluid viscosities [inset in Fig. 2(a)]. We then calculate the contact time $T$ by dividing $L$ with the 


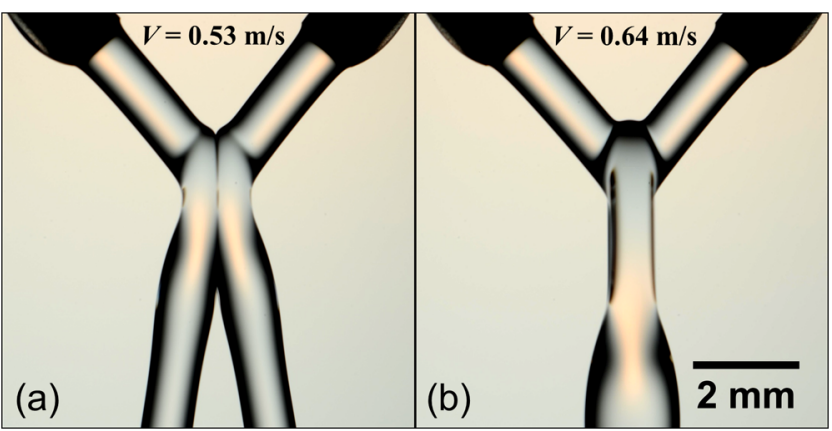

FIG. 1 (color online). (a) Two silicone oil jets (kinematic viscosity $17.9 \mathrm{cSt}$, diameter $0.97 \mathrm{~mm}$, velocity $0.53 \mathrm{~m} / \mathrm{s}$, angle $45^{\circ}$ with the vertical) collide obliquely and bounce off each other and (b) the jets coalesce when the velocity is increased to $0.64 \mathrm{~m} / \mathrm{s}$.

vertical component of the jet velocity, such that $T=$ $L / V \cos \alpha$. Assuming that the rebound of the jets is inertial, $T$ can be derived from a scaling analysis by considering a balance between the kinetic and surface energies at maximal deformation. If the kinetic energy due to velocity normal to the collision plane (of the order of $\rho(V \sin \alpha)^{2} D^{3}$, with $V \sim \delta / T$ where $\delta$ is the change in diameter of the jet during the collision) is balanced with surface energy (of the order of $\sigma \delta^{2}$ ), then we get $T \sim\left(\rho D^{3} \sin ^{2} \alpha / \sigma\right)^{1 / 2}$. In a dimensionless form, this scaling can be written as $T \times V / D \sim(\mathrm{We})^{1 / 2}$, where We is the normal Weber number, defined as $\mathrm{We}=\rho(V \sin \alpha)^{2} D / \sigma$.

Plotted against We on a logarithmic scale, the dimensionless contact time $T \times V / D$ is seen to scale with We, as shown in Fig. 2(a). The best fit line has a slope of 0.49 (rmse $=0.12, R^{2}=0.82$ ), suggesting a square root scaling. The plot includes data for five different values of $D$ and four different values of $\nu$, for various jet angles (see Supplemental Material [21] for the data points corresponding to different angles). Two data points for glycerol solution (which has a much higher surface tension than silicone oil) also fall on top of the silicone oil data, thus confirming the scaling. By rearranging the terms in this scaling, we get $T / D^{3 / 2} \sin \alpha \sim(\rho / \sigma)^{1 / 2}$. For the experiments with different silicone oils, which all have almost the same value of surface tension and density, $T / D^{3 / 2} \sin \alpha$ should be independent of $V$ and roughly constant. Figure 2(b) shows $T / D^{3 / 2} \sin \alpha$ plotted against the $V$, and it shows no significant trend with $V$ (see Supplemental Material [21] for a plot highlighting the effect of diameter).

These results are in good agreement with the previous studies involving drops bouncing on hydrophobic surfaces [22], beads bouncing on elastic membranes [23], and drops bouncing on soap films [17]. The rebound of the jets is caused by surface tension acting like a compressed spring during the collision. Bouncing jets thus act like a classic inertial spring system, for which the time period is proportional to the square root of the ratio of inertia to the spring
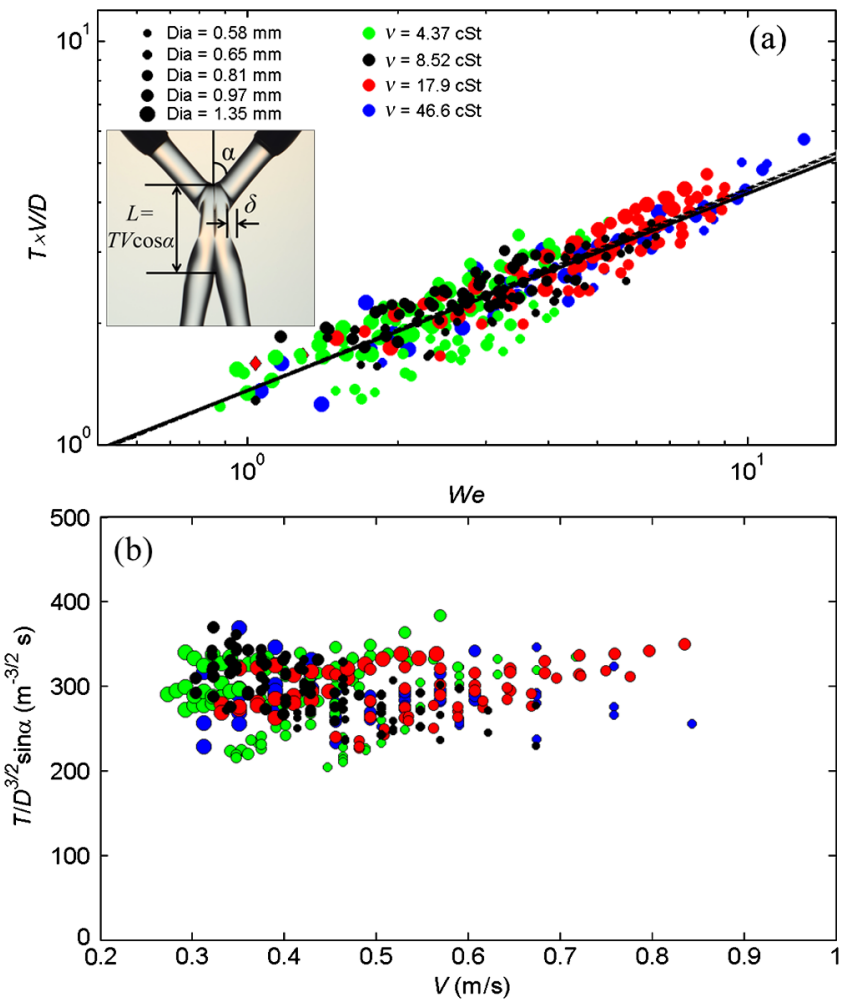

FIG. 2 (color online). (a) log-log plot between dimensionless contact time against normal Weber number (We) for $\nu=4.37 \mathrm{cSt}$ (green), $\nu=8.52 \mathrm{cSt}$ (black), $\nu=17.9 \mathrm{cSt}$ (red), $\nu=46.6 \mathrm{cSt}$ (blue) and $75 \%$ by weight glycerol solution (red diamonds). Solid line is the best fit on the data with slope $=0.49$ (rmse $=$ 0.12 ) and $R^{2}=0.82$. Dashed line is a guide to the eye with a slope of 0.5. Inset: A typical image from the experiment. $L$ is the length of the contact region, $\alpha$ the angle the jets make with the vertical, and $\delta$ is the change in diameter of the jet during the collision. (b) $T / D^{3 / 2} \sin \alpha$ for all silicone oil data indicating velocity independence. Color code is the same as that in (a).

constant. Surface tension acts as the spring constant and the ratio of inertia to the surface tension is given by We. The contact time is, therefore, found to scale with the square root of We, within experimental error.

We investigate the transition of colliding jets from bouncing to coalescence in another set of experiments. In the coalescence state, no bouncing is observed in the jets upon collision. In contrast, the bouncing state is bistable; the jets can coalesce due to dirt or perturbation and stay coalesced. In order to distinguish between such events of coalescence and true transition to coalescence, we employ the following method. Starting with two coalesced jets, bouncing is initiated by perturbing them with a needle and the time elapsed from the beginning of bouncing to recoalescence is measured. This is repeated 20 times and median bouncing life-time and the mean absolute deviation is calculated. Keeping other parameters fixed, the median bouncing life-time of the jets decreases monotonically with an increase in velocity, as shown in the inset in Fig. 3(a). 

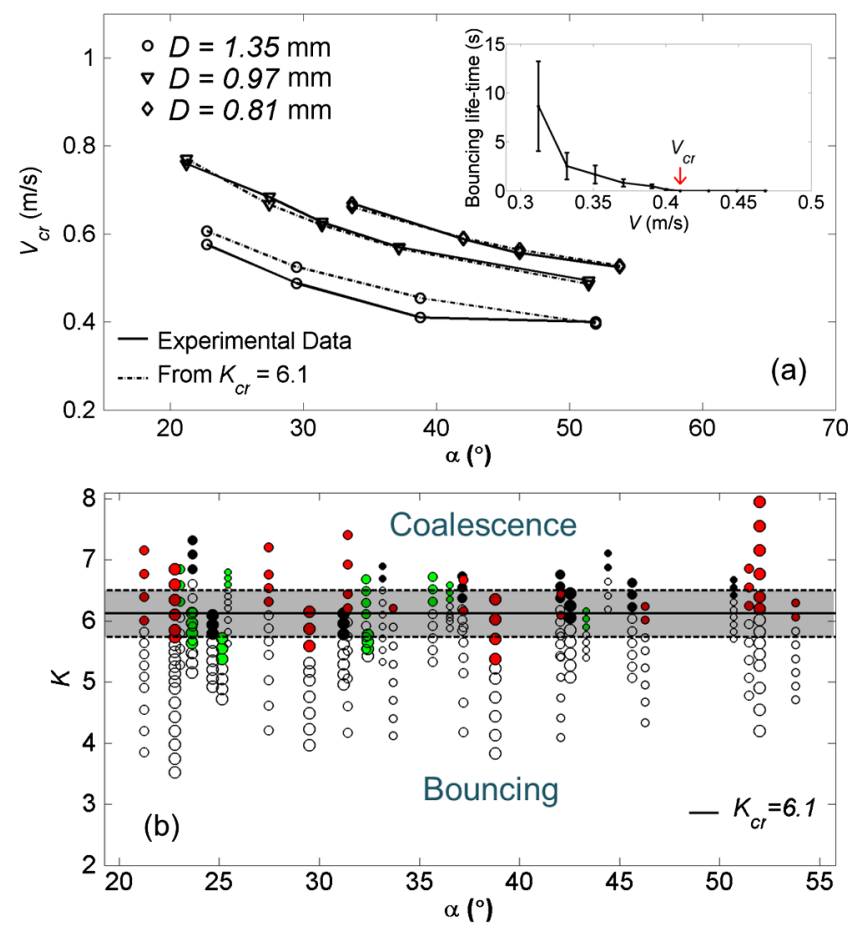

FIG. 3 (color online). (a) Transition velocity $V_{\text {cr }}$ versus $\alpha$ for three different diameters $(\nu=17.9 \mathrm{cSt})$. Solid lines represent experimental data and dashed lines represent transition velocity calculated from $K_{c r}=6.1$. (Inset) Median bouncing life-time versus jet velocity $V$ for $\nu=17.9 \mathrm{cSt}, D=1.35 \mathrm{~mm}, \alpha=38.8^{\circ}$. Red arrow indicates $V_{\text {cr }}$. Error bars are of length equal to one mean absolute deviation. (b) $K=(\mathrm{We} \sqrt{\mathrm{Re}} / \sin \alpha)^{1 / 2}$ versus jet angle $\alpha$. Open symbols represent bouncing and solid symbols represent coalescence. Solid horizontal line represents the $K_{\mathrm{cr}}=6.1$, while the shaded region is of thickness equal to twice the standard deviation $\left(V_{\mathrm{cr}}=0.4, n=32\right.$ ). Color and size code for the symbols is same as that in Fig. 2.

Beyond a threshold velocity, no bouncing is observed in 20 trials and the median bouncing life-time thus dropped down to 0 . This velocity is termed as the transition velocity $\left(V_{\mathrm{cr}}\right)$ for the given set of fluid properties, jet diameter, and angle. For fixed $\nu$, the transition velocity decreases both with an increase in $D$ and in $\alpha$ [Fig. 3(a)]. We thus determined $V_{\text {cr }}$ for various combinations of $\nu, D$, and $\alpha$.

A unifying criterion for transition from bouncing to coalescence is found in terms of the parameter $K$, defined as $K=(\mathrm{We} \sqrt{\operatorname{Re}} / \sin \alpha)^{1 / 2}$, where We is the normal Weber number as defined earlier, and Re the normal Reynolds number defined as $\operatorname{Re}=V \sin \alpha \times D / \nu$. This is graphically shown in Fig. 3(b), which shows $K$ for observations from experiments with four different jet diameters and three different viscosities, with solid symbols representing coalescence and open symbols representing bouncing. The solid, horizontal line represents the average critical value of $K$, termed $K_{\mathrm{cr}}$, which is found to be 6.1. When the transition velocity $V_{\text {cr }}$ is backcalculated from $K_{\text {cr }}=6.1$, it matches very well with the observed transition velocity [dashed lines in Fig. 3(a)].

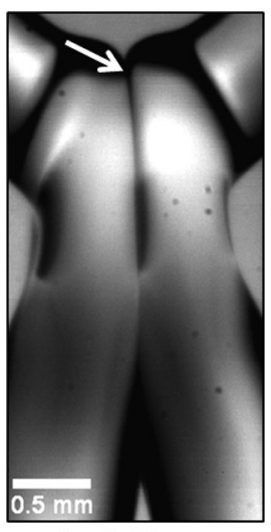

$t=0.15 \mathrm{~ms}$
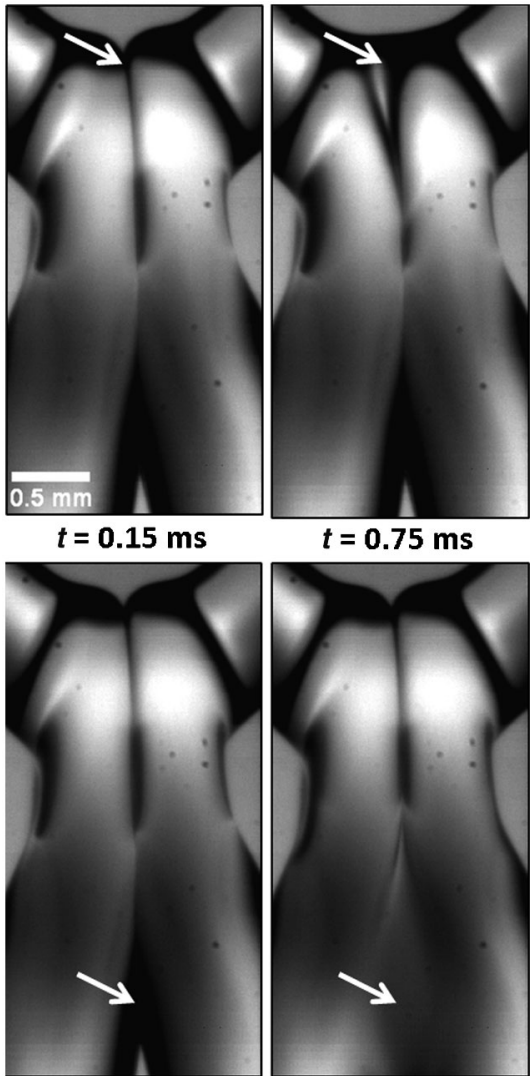

$t=0.75 \mathrm{~ms}$
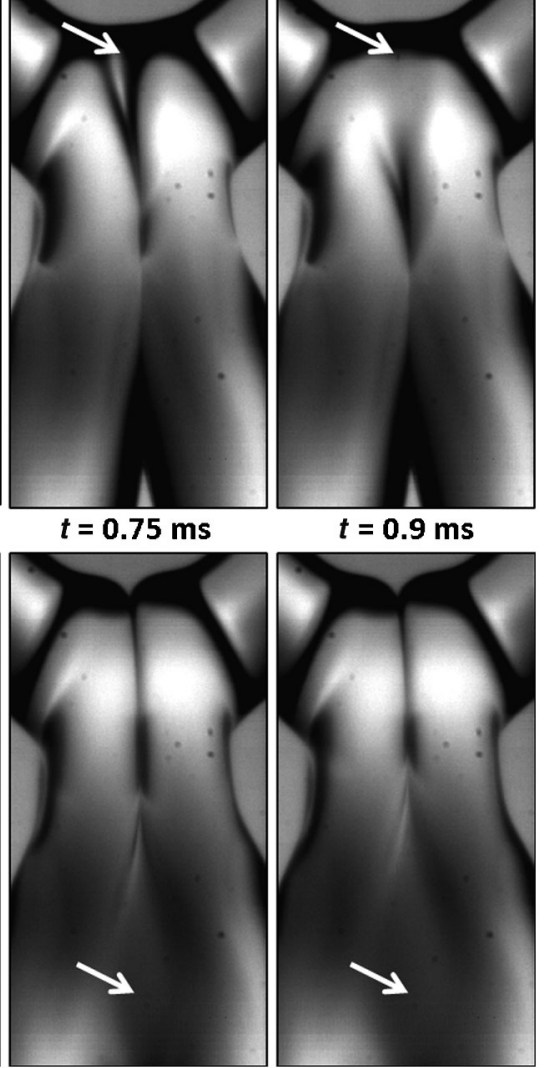

$t=0.9 \mathrm{~ms}$

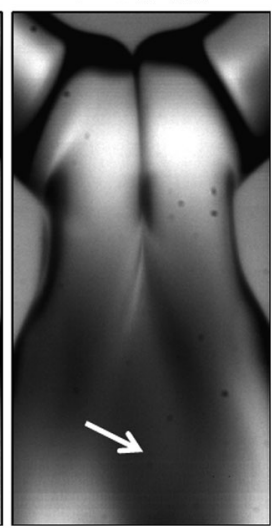

FIG. 4. Transition from bouncing to coalescence. Sequence of high speed images shows coalescence triggering in the top portion of the contact region (top panel) and coalescence triggering in the bottom portion of the contact region (bottom panel). Videos were taken at 20000 frames per second. White arrows point to the location where coalescence occurs. $\nu=17.9 \mathrm{cSt}$, $V=0.59 \mathrm{~m} / \mathrm{s}, D=0.81 \mathrm{~mm}, \alpha=62.7^{\circ}$.

The parameter $K$ has previously been reported in the literature, albeit without the $\sin \alpha$ in the denominator [24-26]. In the phenomenon of drop impact on a solid surface, it has been shown to be responsible for the transition between depositing and crown formation in the impacting drop. We infer that the transition to coalescence in colliding jets is governed by a similar instability as that in the crowning or splashing of drops. During the collision of the jets, at higher velocities, the fluid interface has a tendency to become unstable due to the high value of inertia dominating the surface tension forces. Further, the size of these instabilities or lamellae is dictated by the momentum boundary layer thickness, as argued in the droplet splashing studies [24,27,28]. The balance between inertia and surface tension, combined with the length scale proportionality to the momentum boundary layer thickness, results in the combination of dimensionless numbers denoted as the parameter $K$.

Another possible explanation for the transition from bouncing to coalescence is the thinning of the air film separating the jets, to a critical thickness required for 
coalescence [15]. The thickness of the air film is expected to be minimum at the midpoint of the contact region, as it is at the midpoint where the jets start to recoil. When this minimum thickness becomes equal to the critical thickness required for van der Waals forces to become dominant, the jets coalesce. The thickness at which van der Waals forces become important has been reported to be around $100-200 \mathrm{~nm}[13,15,17]$. If coalescence is caused by thinning of the air film to this critical thickness, we should expect it to always start at the midpoint of the contact region. If an instability on the interface is responsible for the coalescence, it could start at any point in the contact region, though the midpoint is still a more likely location due to the smaller thickness of the air film.

We used high speed imaging to film the contact region between the jets at the time of coalescence. Figure 4 shows high-speed images from two different events of coalescence between two silicone oil jets under the same experimental conditions (see Supplemental Material [21] for high speed movies). The top panel shows a case in which coalescence is triggered in the top portion of the contact region, while the images from another coalescence incident (bottom panel) show that coalescence is triggered in the bottom portion of the contact region. We also see other cases in which coalescence is triggered in other locations in the contact region. From this evidence, we conclude that an instability on the interface of colliding jets is responsible for a transition to coalescence, the quantitative criterion for which is given by the parameter $K$, described above.

In summary, we have presented the first quantitative investigation on the noncoalescence between jets of the same fluid. Our experiments show that the recoil of the jets after the collision is surface-tension dominated, an implication of which is that the dimensionless contact time of bouncing jets scales with the normal Weber number (We). Further, a parametric investigation of the transition from bouncing to coalescence in colliding jets shows that the parameter $K=(\mathrm{We} \sqrt{\operatorname{Re}} / \sin \alpha)^{1 / 2}$ governs whether the jets will bounce or coalesce upon collision. The emergence of $K$ as the criterion for transition to coalescence suggests that jet coalescence is caused by a splashing-type instability on the fluid-air interface.

Bouncing jets is a rich system to study; while we focused on the collision of jets with identical properties, one could also have different hydrodynamic and physical properties in the two colliding jets, with a different set of dimensionless parameters for each of the jets. We also observed bouncing in three colliding jets for two different configurations, one in which jets were in the same vertical plane and another in which they were in three different planes (see Supplemental Material [21] for images). We hope that the stable and continuous noncoalescence in bouncing jets can be useful in gaining a better understanding of fluidfluid noncoalescence and interfacial dynamics.
Authors wish to thank Tomas Bohr and Anders Andersen for useful discussions. Acknowledgment is made to the donors of American Chemical Society Petroleum Research Fund No. (PRF\# 52332-DNI9) for support of this research.

*Present address: Department of Physics, Technical University of Denmark, 2800 Kongens Lyngby, Denmark. †sunnyjsh@vt.edu

[1] P. Shah, L. Fan, I. Kao, and L. Erickson, Advances in Applied Microbiology (Academic Press, New York, 1972), Vol. 15, p. 367.

[2] P. Simpkins and V. Kuck, J. Colloid Interface Sci. 263, 562 (2003).

[3] P. Calvert, Chem. Mater. 13, 3299 (2001).

[4] K.-Y. Lee, H.-G. Lee, and P. C. Hayes, ISIJ International (1989-) (Iron and Steel Institute of Japan) 38, 1233 (1998).

[5] L. Rayleigh, Proc. R. Soc. London 28, 405 (1878).

[6] L. Rayleigh, Philos. Mag. 48, 321 (1899).

[7] S. G. Bradley and C. D. Stow, Phil. Trans. R. Soc. A 287, 635 (1978).

[8] Y. J. Jiang, A. Umemura, and C. K. Law, J. Fluid Mech. 234, 171 (1992).

[9] J. Qian and C. K. Law, J. Fluid Mech. 331, 59 (1997).

[10] M. Thrasher, S. Jung, Y. K. Pang, C.-P. Chuu, and H. L. Swinney, Phys. Rev. E 76, 056319 (2007).

[11] O. Reynolds, Memoirs and Proceedings/Manchester Literary and Philosophical Society 21, 1 (1881).

[12] L. Mahajan, Nature (London) 126, 761 (1930).

[13] G. Charles and S. Mason, J. Colloid Sci. 15, 236 (1960).

[14] G. Neitzel and P. Dell'Aversana, Annu. Rev. Fluid Mech. 34, 267 (2002).

[15] Y. Couder, E. Fort, C.-H. Gautier, and A. Boudaoud, Phys. Rev. Lett. 94, 177801 (2005).

[16] L. Courbin and H. A. Stone, Phys. Fluids 18, 091105 (2006).

[17] T. Gilet and J. W. M. Bush, J. Fluid Mech. 625, 167 (2009).

[18] K. R. Sreenivas, P. K. De, and J. H. Arakeri, J. Fluid Mech. 380, 297 (1999).

[19] N. Wadhwa and S. Jung, Phys. Fluids 23, 091105 (2011).

[20] G. Batchelor, An Introduction to Fluid Dynamics (Cambridge University Press, Cambridge, 2000).

[21] See Supplemental Material at http://link.aps.org/ supplemental/10.1103/PhysRevLett.110.124502 for additional plots, images of three bouncing jets, and high speed movies of coalescence of two jets.

[22] D. Richard, C. Clanet, and D. Quéré, Nature (London) 417, 811 (2002).

[23] L. Courbin, A. Marchand, A. Vaziri, A. Ajdari, and H. A. Stone, Phys. Rev. Lett. 97, 244301 (2006).

[24] A. L. Yarin and D. A. Weiss, J. Fluid Mech. 283, 141 (1995).

[25] C. D. Stow and M. G. Hadfield, Proc. R. Soc. A 373, 419 (1981).

[26] C. Mundo, M. Sommerfeld, and C. Tropea, Int. J. Multiphase Flow 21, 151 (1995).

[27] I. V. Roisman, K. Horvat, and C. Tropea, Phys. Fluids 18, 102104 (2006).

[28] J. C. Bird, S. S. H. Tsai, and H. A. Stone, New J. Phys. 11, 063017 (2009). 